\title{
Wealth Stratification and Portfolio Choice
}

\author{
Karl David Boulware \\ Kenneth N. Kuttner* \\ January 16, 2020
}

\section{ONLINE APPENDIX}

\footnotetext{
*Boulware: kboulware@ wesleyan.edu. Department of Economics, Wesleyan University, 238 Church Street, Middletown, CT, 06459, USA; Kuttner: kenneth.n.kuttner@williams.edu. Department of Economics, Williams College, 24 Hopkins Hall Drive, Williamstown, MA 01267, USA.
} 


\section{Appendix Table 1}

Descriptive statistics by asset decile

\begin{tabular}{lrrrrrrrrrrr}
\hline & \multicolumn{10}{c}{ Asset decile } & \\
\cline { 2 - 10 } & 1 & 2 & 3 & 4 & 5 & 6 & 7 & 8 & 9 & 10 & Average \\
\hline Assets, \$1000s & 0 & 2 & 14 & 64 & 129 & 206 & 303 & 469 & 902 & 5546 & 763 \\
Net worth, \$1000s & -9 & -12 & -8 & 33 & 69 & 109 & 191 & 337 & 728 & 5243 & 668 \\
Wage income, \$1000s & 14 & 22 & 35 & 34 & 38 & 49 & 55 & 61 & 90 & 218 & 62 \\
White share, \% & 43 & 47 & 58 & 60 & 69 & 73 & 80 & 78 & 84 & 87 & 68 \\
Black share, \% & 29 & 29 & 21 & 22 & 18 & 13 & 11 & 9 & 5 & 2 & 16 \\
Hispanic share, \% & 24 & 18 & 15 & 12 & 12 & 10 & 7 & 8 & 4 & 2 & 11 \\
\hline
\end{tabular}

The figures are the averages for each decile. Assets and net worth exclude the value of vehicles. The racial/ethnic shares do not sum to 100, due to the omission of the "other" and "decline to state" categories. The data are from the 2016 wave of the Survey of Consumer Finances.

\section{Appendix Table 2}

Portfolio allocation by wealth decile and race/ethnicity

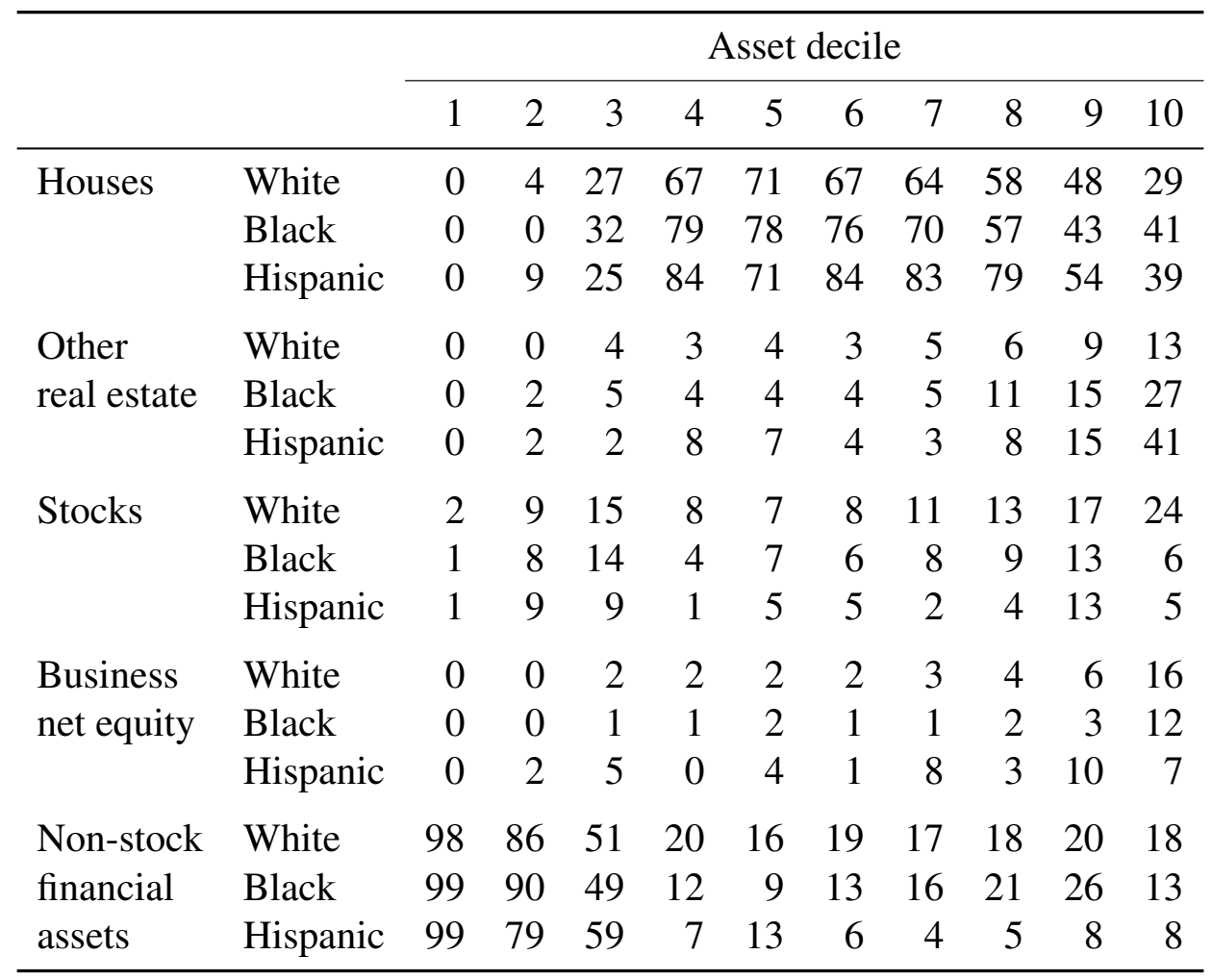

Notes: The figures are the shares of total assets (excluding vehicles) allocated to each of the indicated asset categories, expressed in percentage terms. Shares may not sum to 100 due to rounding. The data are from the 2007 wave of the Survey of Consumer Finances. 


\section{Appendix Table 3}

Results from portfolio share regressions

\begin{tabular}{|c|c|c|c|c|c|}
\hline & Houses & Stocks & Business & Other RE & Other financial \\
\hline Log assets & $\begin{array}{c}9.59^{* * *} \\
(0.26)\end{array}$ & $\begin{array}{c}0.75^{* * *} \\
(0.076)\end{array}$ & $\begin{array}{c}-0.21^{* * *} \\
(0.060)\end{array}$ & $\begin{array}{c}0.091 \\
(0.057)\end{array}$ & $\begin{array}{c}-10.2^{* * *} \\
(0.19)\end{array}$ \\
\hline Log assets squared & $\begin{array}{c}-0.59^{* * *} \\
(0.034)\end{array}$ & $\begin{array}{l}0.044^{* * *} \\
(0.012)\end{array}$ & $\begin{array}{c}0.28^{* * *} \\
(0.010)\end{array}$ & $\begin{array}{c}0.19^{* * *} \\
(0.0098)\end{array}$ & $\begin{array}{c}0.073^{* *} \\
(0.025)\end{array}$ \\
\hline Black/AA & $\begin{array}{c}-0.82 \\
(1.48)\end{array}$ & $\begin{array}{c}-0.72 \\
(0.64)\end{array}$ & $\begin{array}{r}-0.64^{*} \\
(0.26)\end{array}$ & $\begin{array}{c}0.37 \\
(0.39)\end{array}$ & $\begin{array}{c}1.81 \\
(1.25)\end{array}$ \\
\hline Black $\times \log$ assets & $\begin{array}{c}1.02^{* *} \\
(0.33)\end{array}$ & $\begin{array}{c}-0.59^{* * *} \\
(0.11)\end{array}$ & $\begin{array}{l}-0.12 \\
(0.077)\end{array}$ & $\begin{array}{l}0.47^{* * *} \\
(0.12)\end{array}$ & $\begin{array}{c}-0.78^{* *} \\
(0.25)\end{array}$ \\
\hline Hispanic & $\begin{array}{c}-6.89^{* * *} \\
(1.79)\end{array}$ & $\begin{array}{c}1.28 \\
(0.79)\end{array}$ & $\begin{array}{c}0.48 \\
(0.46)\end{array}$ & $\begin{array}{c}-0.21 \\
(0.46)\end{array}$ & $\begin{array}{l}5.34^{* * *} \\
(1.61)\end{array}$ \\
\hline Hispanic $\times \log$ assets & $\begin{array}{l}1.81^{* * *} \\
(0.37)\end{array}$ & $\begin{array}{c}-1.00^{* * *} \\
(0.13)\end{array}$ & $\begin{array}{c}-0.12 \\
(0.14)\end{array}$ & $\begin{array}{l}0.88^{* * *} \\
(0.16)\end{array}$ & $\begin{array}{c}-1.56^{* * *} \\
(0.28)\end{array}$ \\
\hline Other & $\begin{array}{c}0.43 \\
(1.32)\end{array}$ & $\begin{array}{c}-1.90^{* *} \\
(0.71)\end{array}$ & $\begin{array}{c}-0.73 \\
(0.49)\end{array}$ & $\begin{array}{c}0.34 \\
(0.56)\end{array}$ & $\begin{array}{c}1.87 \\
(0.99)\end{array}$ \\
\hline Age & $\begin{array}{l}9.07^{* * *} \\
(0.96)\end{array}$ & $\begin{array}{c}1.29^{*} \\
(0.54)\end{array}$ & $\begin{array}{c}-1.23^{* * *} \\
(0.36)\end{array}$ & $\begin{array}{r}-1.04^{*} \\
(0.42)\end{array}$ & $\begin{array}{c}-8.08^{* * *} \\
(0.82)\end{array}$ \\
\hline Age squared & $\begin{array}{c}-0.81^{* * *} \\
(0.088)\end{array}$ & $\begin{array}{c}-0.18^{* * *} \\
(0.049)\end{array}$ & $\begin{array}{c}0.021 \\
(0.032)\end{array}$ & $\begin{array}{c}0.094^{*} \\
(0.041)\end{array}$ & $\begin{array}{c}0.87^{* * *} \\
(0.076)\end{array}$ \\
\hline Children & $\begin{array}{c}3.06^{* * *} \\
(0.27)\end{array}$ & $\begin{array}{c}-1.45^{* * *} \\
(0.13)\end{array}$ & $\begin{array}{c}-0.068 \\
(0.12)\end{array}$ & $\begin{array}{c}0.045 \\
(0.12)\end{array}$ & $\begin{array}{c}-1.59^{* * *} \\
(0.20)\end{array}$ \\
\hline Education & $\begin{array}{c}-2.86^{* * *} \\
(0.11)\end{array}$ & $\begin{array}{c}1.17^{* * *} \\
(0.056)\end{array}$ & $\begin{array}{c}-0.31^{* * *} \\
(0.047)\end{array}$ & $\begin{array}{c}-0.11 \\
(0.058)\end{array}$ & $\begin{array}{c}2.10^{* * *} \\
(0.082)\end{array}$ \\
\hline Married & $\begin{array}{r}-1.25^{*} \\
(0.61)\end{array}$ & $\begin{array}{c}0.18 \\
(0.34)\end{array}$ & $\begin{array}{c}-0.22 \\
(0.22)\end{array}$ & $\begin{array}{l}1.03^{* * *} \\
(0.26)\end{array}$ & $\begin{array}{c}0.26 \\
(0.47)\end{array}$ \\
\hline Constant & $\begin{array}{l}21.6^{* * *} \\
(2.87)\end{array}$ & $\begin{array}{c}-4.50^{* *} \\
(1.62)\end{array}$ & $\begin{array}{l}5.49^{* * *} \\
(1.02)\end{array}$ & $\begin{array}{c}0.88 \\
(1.17)\end{array}$ & $\begin{array}{l}76.5^{* * *} \\
(2.34)\end{array}$ \\
\hline$R^{2}$ & 0.277 & 0.085 & 0.096 & 0.064 & 0.527 \\
\hline
\end{tabular}

Notes: The dependent variable is the share of assets allocated to the categories given in the column headers, expressed as a percent. The education variable represents the highest degree earned: $8=$ high school graduate, $9=$ some college but no degree, $10=$ associate degree in a vocational or occupational program, and $12=$ bachelor's degree. Age is expressed in decades. Heteroskedasticityconsistent standard errors are in parentheses. Asterisks denote statistical significance: ${ }^{*} p<0.05$, ${ }^{* *} p<0.01,{ }^{* * *} p<0.001$. The number of observations is 21,240 in all regressions. The data are from the 2007 wave of the Survey of Consumer Finances. 